\title{
Sequential occurrence of recurrent Sweet syndrome and erythema nodosum without an underlying secondary cause: a case report
}

\author{
Chamila Mettananda ${ }^{{ }^{*}} \mathbb{0}$, Hansika Peiris ${ }^{1}$ and Ahamed Uwyse ${ }^{2}$
}

\begin{abstract}
Background: Sweet syndrome is a rare cause of acute fever and painful erythematous skin plaques. Erythema nodosum is acute or chronic tender erythematous skin nodules of bilateral shins. The concurrent presence of both dermatoses is rare but reported in the literature. There are no reported cases of recurrent and sequential Sweet syndrome and erythema nodosum without an underlying secondary cause.

Case presentation: We report the case of a 64-year-old Asian woman, who had possible Sweet syndrome 12 years ago and biopsy-proven erythema nodosum 5 years ago, presenting with an acute episode of Sweet syndrome. Extensive investigations did not reveal any underlying secondary cause.

Conclusions: Recurrent Sweet syndrome and sequential presence with erythema nodosum raises suspicion if Sweet syndrome and erythema nodosum are different presentations of one disease, which warrants further study. This case proves that recurrent Sweet syndrome and erythema nodosum can occur in healthy individuals without an underlying malignancy or secondary cause.
\end{abstract}

Keywords: Fever, Rash, Sweet syndrome, Erythema nodosum red-eye, Case report

\section{Background}

Sweet syndrome (SS) is an acute febrile neutrophilic dermatosis. It is a rare differential diagnosis in a patient with fever, usually presenting as fever and acute painful erythematous skin plaques, arthralgia, and episcleritis $[1,2]$. SS presenting following infections or an immunological cause is called classic Sweet syndrome. It can also be malignancy induced [3, 4] or drug induced [2]. Pathologically, there is edema on the upper dermis and intense neutrophilic infiltration [2]. Recurrent Sweet syndrome is reported in about one-third of patients [2].

*Correspondence: chamila@kln.ac.lk

1 Department of Pharmacology, Faculty of Medicine, University of Kelaniya, Ragama, Sri Lanka

Full list of author information is available at the end of the article
Erythema nodosum (EN) is acute or chronic tender erythematous skin nodules of bilateral shins [5]. It has been shown to follow streptococcal pharyngitis, but may precede tuberculosis, bacterial or deep fungal infection, sarcoidosis, inflammatory bowel disease, or cancer; however, it is mainly idiopathic. Pathologically, it shows granulomatous panniculitis [6,7].

The concurrence of both dermatoses is rare but reported in the literature: eight cases without underlying malignancies [8], one case following sarcoidosis [9], one case following Crohn's disease [10], two cases following streptococcal upper respiratory infections $[9,11]$, and one case following acute myeloid leukemia [12]. Both are reactive dermatoses, but the pathogenesis is not well understood [13-15]. 
Sequential presence of SS and then EN is even rarer; there is one case reported in a patient with myeloid leukemia [16]. Our case is the first to report sequential presentation of SS and EN without an underlying malignancy or secondary cause.

\section{Case presentation}

A 64-year old, previously healthy, Asian woman presented with a 4-day history of fever, and multiple, between 1 and $3 \mathrm{~cm}$ in width, irregularly shaped, tender, red-color, palpable, non-scaly skin plaques over dorsal aspects of knees, elbows, and shins with ankle joint pain and left-sided painless red eye (Fig. 1). There was no clinical proximal muscle weakness or racoon eyes. The rest of the examination was normal. Initially, an infectious etiology was suspected. She had high inflammatory markers, erythrocyte sedimentation rate (ESR) $117 \mathrm{~mm}$ after 1 hour, C-reactive protein (CRP) $168 \mathrm{mg} / \mathrm{dl}$, white blood cells $12.5 \times 10^{3} / \mu \mathrm{L}$, neutrophils $67 \%$, but a negative septic screen, and creatine phosphokinase (CPK) $72 \mathrm{U} / \mathrm{L}$. She was promptly started on intravenous co-amoxiclav and oral ciprofloxacin to cover an infection. On further questioning, she had had a fever with a similar skin rash, involving upper arms and legs, treated in hospital for about a week 12 years ago for which the records were not available. However, she was treated for biopsy-proven erythema nodosum 5 years ago, which settled without any consequences. With this past medical history, Sweet syndrome was suspected, and she was started on oral methylprednisolone $16 \mathrm{mg}$ daily while being on antibiotics. The symptoms improved dramatically in 2 days, and skin biopsy was not performed as the patient refused it with quick symptom resolution. However, diagnostic criteria were fulfilled and were pointing to a diagnosis of Sweet syndrome. The plaques disappeared completely, leaving only skin discoloration by 1 week (Fig. 2). Inflammatory markers also improved significantly by 1 week; ESR $60 \mathrm{~mm}$ after 1 hour and CRP $8 \mathrm{mg} / \mathrm{dl}$. The patient was treated with methylprednisolone $16 \mathrm{mg}$ daily for 2 weeks, and it was tailed off over 6 weeks. A comprehensive set of investigations including liver biochemistry, serum amylase, blood picture, chest X-ray, and ultrasound (US) abdomen did not find any underlying secondary cause for the recurrent SS and EN in this patient. The patient was followed up 9 months after the current presentation, and she was asymptomatic with an ESR of $20 \mathrm{~mm}$ after 1 hour.

\section{Discussion}

This patient had classic symptoms of Sweet syndrome involving skin, eyes, and joints. Laboratory investigations were supportive of the diagnosis and excluded other differential diagnoses. Swift response of the disease to steroids helped the diagnosis. She did not have any evidence of underlying malignancy, nor was she on any medication known to precipitate Sweet syndrome. Therefore, classic Sweet syndrome was diagnosed on criteria [17]. We did not find any precipitant for classic SS such as respiratory infection, or other inflammatory diseases. She had EN 5 years ago. The first presentation 12 years ago was also likely to be SS but no skin biopsy had been done to confirm.

The concurrent presence of SS and EN is reported in the literature [8-12]. Sequential presence of SS and EN is reported in a patient with myeloid leukemia [16]. This is the first case reported of sequential presentation of SS and EN in an otherwise healthy individual without any underlying secondary cause, observed over 12 years duration.
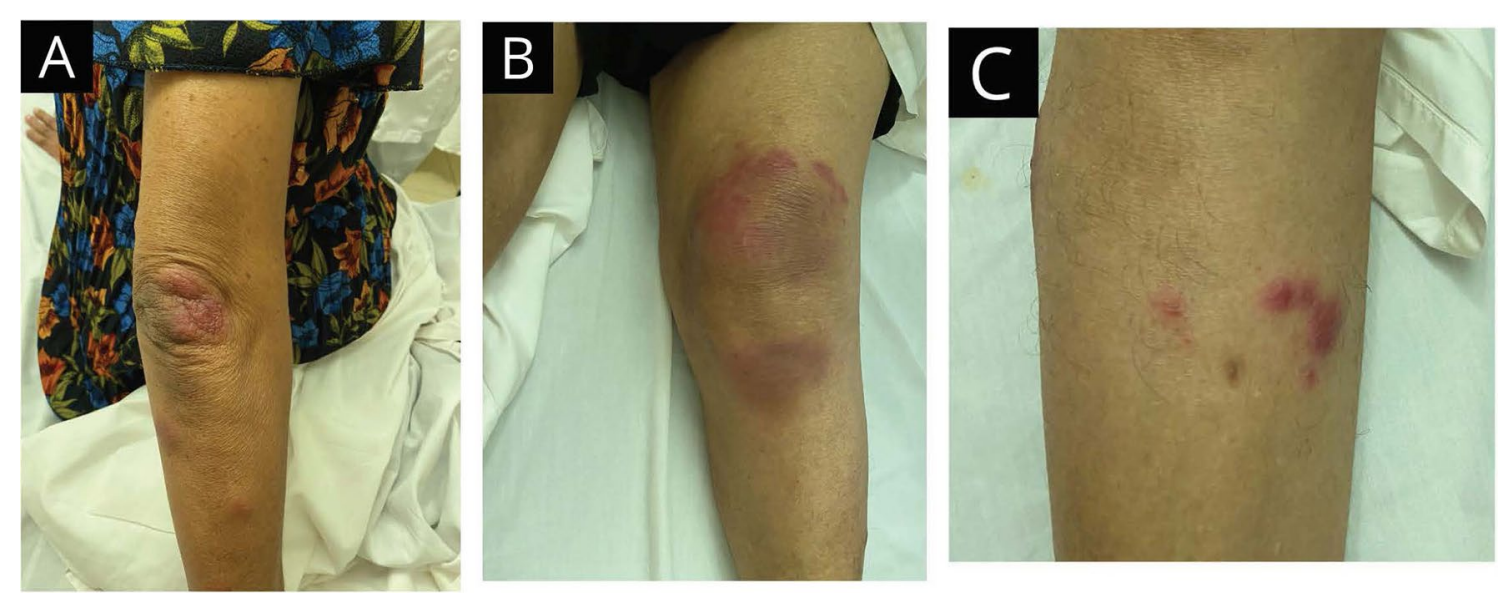

Fig. 1 At presentation—4 days after onset of fever and rash. Tender erythematous skin plaques over (A) right elbow joint, (B) left knee joint, and (C) right shin 


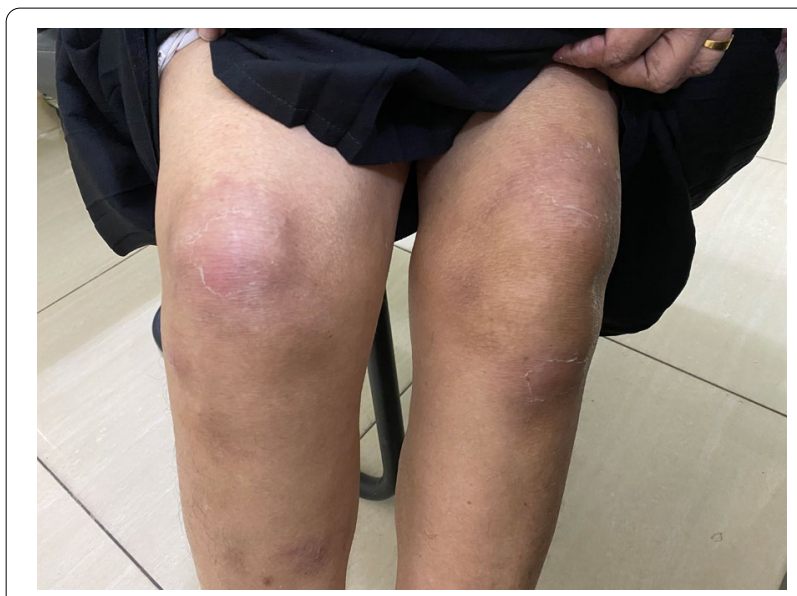

Fig. 2 After treatment-14 days after the onset of illness. Healing skin rash without scarring

The possible recurrent nature of SS and sequential presence of EN need further investigation into the pathogenesis of the disease to rule out if this is a spectrum of one disease and not two clinical entities. SS and EN have several features in common: both are reactive dermatoses; the usual stimuli are the same; skin manifestations are very similar with the only difference being in the distribution of the skin lesions, with EN usually being confined to the dorsal aspect of lower limbs; and both respond to the same treatment, with steroids, either oral or topical, being useful in the management of these skin disorders [18, 19]. We considered our patient at the current presentation as having concurrent SS and EN, yet a misdiagnosis is possible, as she may have had SS only, with some features of EN. However, the suspicion for it being a spectrum of one same disease is even higher when SS and EN present sequentially, which does not give room for misdiagnosis EN. In SS, there is a dense, mature, neutrophilic infiltration of the upper dermis $[2,20,21]$. EN shows a neutrophilic infiltrate in the early stage, and though histological presentation can vary, its hallmark is granulomatous panniculitis called Miescher's radial granulomas $[5,6]$. EN is reported to show lobular neutrophilic panniculitis with suppuration, small-vessel vasculitis, and even medium-vessel arteritis, although rarely [22, 23].

A minority of cases with SS and EN can be associated with malignancies. Around $16 \%$ of Sweet syndrome cases are malignancy related and can precede, follow, or appear concurrent with the diagnosis of the patient's neoplasm, especially hematological malignancies [4]. Many cases of EN are idiopathic and less associated with malignancies compared with SS $[5,6]$. EN can be a cutaneous marker of lymphoma/leukemia [24, 25] and carcinoid/colorectal/pancreatic cancers $[26,27]$ and can be a marker of recurrence of those malignancies. However, the presence of recurrent SS and EN in the same patient without an underlying secondary cause, as we reported, suggests that patients may have recurrent SS or concurrent/sequential $\mathrm{SS}$ and EN that is not always indicative of an underlying chronic disease or a malignancy; instead, it could be idiopathic.

\section{Conclusions}

There are no reported cases of recurrent and sequential SS and EN without an underlying secondary cause. We report a 64-year-old, otherwise healthy, Asian woman with sequential recurrent SS and EN over 12 years without an underlying secondary cause. Recurrent SS and sequential presence with EN raise the suspicion of SS and EN being different presentations of one disease, which warrants further study. This case proves that recurrent SS and sequential SS and EN may occur in healthy individuals without an underlying malignancy or secondary cause.

\section{Acknowledgements}

None.

\section{Authors' contributions}

All authors contributed to the writing of this report. KCDM wrote and prepared the first draft of the manuscript, with input from the writing committee, all of whom approved the decision to submit the manuscript for publication. All authors read and approved the final manuscript.

\section{Funding}

Not applicable.

\section{Availability of data and materials \\ Not applicable.}

\section{Declarations}

Ethics approval and consent to participate Not applicable.

\section{Consent for publication}

Written informed consent was obtained from the patient for publication of this case report and any accompanying images. A copy of the written consent is available for review by the Editor-in-Chief of this journal.

\section{Competing interests}

The authors declare that they have no competing interests.

\section{Author details}

${ }^{1}$ Department of Pharmacology, Faculty of Medicine, University of Kelaniya, Ragama, Sri Lanka. ${ }^{2}$ North Colombo Teaching Hospital, Ragama, Sri Lanka.

Received: 30 September 2021 Accepted: 20 January 2022 Published online: 25 February 2022

\section{References}

1. von den Driesch P. Sweet's syndrome (acute febrile neutrophilic dermatosis). J Am Acad Dermatol. 1994;31(4):535-56 (quiz 57-60).

2. Cohen PR. Sweet's syndrome-a comprehensive review of an acute febrile neutrophilic dermatosis. Orphanet J Rare Dis. 2007;2(1):34. 
3. Cohen PR, Kurzrock R. Sweet's syndrome and cancer. Clin Dermatol. 1993;11(1):149-57.

4. Bourke JF, Keohane S, Long CC, Kemmett D, Davies M, Zaki I, et al. Sweet's syndrome and malignancy in the U.K. Br J Dermatol. 1997;137(4):609-13.

5. Schwartz RA, Nervi SJ. Erythema nodosum: a sign of systemic disease. Am Fam Physician. 2007;75(5):695-700.

6. Chowaniec M, Starba A, Wiland P. Erythema nodosum — review of the literature. Reumatologia. 2016;54(2):79-82.

7. Pérez-Garza DM, Chavez-Alvarez S, Ocampo-Candiani J, Gomez-Flores M. Erythema nodosum: a practical approach and diagnostic algorithm. Am J Clin Dermatol. 2021;22(3):367-78.

8. Cohen PR, Holder WR, Rapini RP. Concurrent Sweet's syndrome and erythema nodosum: a report, world literature review and mechanism of pathogenesis. J Rheumatol. 1992;19(5):814-20.

9. Wilkinson SM, Heagerty AHM, English JSC. Acute febrile neutrophilic dermatosis in association with erythema nodosum and sarcoidosis. Clin Exp Dermatol. 1993;18(1):47-9.

10. Waltz KM, Long D, Marks JG Jr, Billingsley EM. Sweet's syndrome and erythema nodosum: the simultaneous occurrence of 2 reactive dermatoses. Arch Dermatol. 1999:135(1):62-6.

11. Ben-Noun L. Sweet's syndrome associated with erythema nodosum. Aust Fam Physician. 1995;24(10):1867-9.

12. von den Driesch P, Steffan C, Zöbe A, Hornstein OP. Sweet's syndrometherapy with cyclosporin. Clin Exp Dermatol. 1994;19(3):274-7.

13. Tabanlıoğlu D, Boztepe G, Erkin G, Gököz Ö, Karaduman A. Sweet's syndrome and erythema nodosum: a companionship or a spectrum?-a case report with review of the literature. Int J Dermatol. 2010:49(1):62-6.

14. Harris T, Henderson MC. Concurrent Sweet's syndrome and erythema nodosum. J Gen Intern Med. 2011:26(2):214-5.

15. Wasson S, Govindarajan G, Folzenlogen D. Concurrent occurrence of Sweet's syndrome and erythema nodosum: an overlap in the spectrum of reactive dermatoses. Clin Rheumatol. 2006;25(2):268-72.

16. Suzuki Y, Kuroda K, Kojima T, Fujita M, Iseki T, Shiki H. Unusual cutaneous manifestations of myelodysplastic syndrome. Br J Dermatol. 1995;133(3):483-6.

17. Cohen PR, Kurzrock R. Diagnosing the Sweet syndrome. Ann Intern Med. 1989;110(7):573-4.

18. Devaraj NK, Aneesa AR, Abdul Hadi AM, Shaira N. Topical corticosteroids in clinical practice. Med J Malaysia. 2019;74(2):187-9.

19. Devaraj NK. A recurrent cutaneous eruption. BMJ Case Rep. 2019. https:// doi.org/10.1136/bcr-2018-228355

20. Going JJ, Going SM, Myśkoẃ MW, Beveridge GW. Sweet's syndrome: histological and immunohistochemical study of 15 cases. J Clin Pathol. 1987:40(2):175-9.

21. Majmundar VD, Baxi K. Acute Febrile Neutrophilic Dermatosis. StatPearls. Treasure Island (FL): StatPearls Publishing. Copyright $\odot ~ 2021$, StatPearls Publishing LLC.; 2021.

22. Thurber S, Kohler S. Histopathologic spectrum of erythema nodosum. J Cutan Pathol. 2006;33(1):18-26.

23. Winkelmann RK, Förström L. New observations in the histopathology of erythema nodosum. J Invest Dermatol. 1975;65(5):441-6.

24. Sullivan R, Clowers-Webb H, Davis MD. Erythema nodosum: a presenting sign of acute myelogenous leukemia. Cutis. 2005;76(2):114-6.

25. Bonci A, Di Lernia V, Merli F, Lo SG. Erythema nodosum and Hodgkin's disease. Clin Exp Dermatol. 2001;26(5):408-11.

26. Lin JT, Chen PM, Huang DF, Kwang WK, Lo K, Wang WS. Erythema nodosum associated with carcinoid tumour. Clin Exp Dermatol. 2004;29(4):426-7.

27. Durden FM, Variyam E, Chren MM. Fat necrosis with features of erythema nodosum in a patient with metastatic pancreatic carcinoma. Int J Dermatol. 1996;35(1):39-41.

\section{Publisher's Note}

Springer Nature remains neutral with regard to jurisdictional claims in published maps and institutional affiliations.

Ready to submit your research? Choose BMC and benefit from:

- fast, convenient online submission

- thorough peer review by experienced researchers in your field

- rapid publication on acceptance

- support for research data, including large and complex data types

- gold Open Access which fosters wider collaboration and increased citations

- maximum visibility for your research: over $100 \mathrm{M}$ website views per year

At $\mathrm{BMC}$, research is always in progress.

Learn more biomedcentral.com/submissions 